\title{
Alternativas de financiamiento de la atención médica en América Latina y el Caribe
}

\author{
Alternatives for the financing of health care in Latin America and the Caribbean
}

\author{
Antonio Carlos Coelho Campino* \\ Departamento de Economia - Faculdade de Economia, Administração e \\ Contabilidade da Universidade de São Paulo, Brasil
}

\begin{abstract}
Dada la magnitud del problema de salud en los paises de America Latina y el Caribe, el déficit de recursos flnancieros necesarios para proveer atenclón méclca no se puede cubrir a través de aumentos en los precios, o adoptando medidas como pago por servicio o cobro por los costos de los servicios. Es necesario recurrir a la política fiscal. Se demuestra que es posible aumentar los recursosos disponibles para la atención médica, tanto por el aumento en la recaudación de impuestos, como por la toma de decisión política de aumentar los ingresos del gobierno destinados a financiar la atención médica. Se revisa la experiencla de los Fondos de Emergencia Social y de las operaciones de cambio de deuda por salud como Importantes fuentes adicionales de recursos especialmente para financiar la ínversión.
\end{abstract}

Financiamiento de salud. Financiamiento governamental. Gastos en salud.

\section{Introducción}

El tema del financiamiento de la atención médica últimamente ha recibido una atención creciente. Las principales razones para esto son el elevado nivel de gasto realizado por los gobiernos en la atención médica y la tasa positiva de crecimiento que presentan estos gastos. Así, por ejemplo en países como los Estados Unidos, hoy en día, los gastos en salud representan entre el 10 y el $12 \%$ del Producto Nacional Bruto (PNB) y esta proporción tiende a crecer. El crecimiento del gasto en salud a tasas mayores que las tasas de crecimiento del PNB se debe principalmente a la utilización de tecnologías de alta sofisticación, mas costosas, y a la extensión de los seguros de salud.

Los hospitales tienen un rol creciente en el incremento de gastos en salud. Así, Newbrander et al $^{9}$. (1992) observan que los hospitales representan con mucha frecuencia del 50 al $80 \%$ del gasto recurrente del sector salud y utilizan aproximadamente la mitad del dispendio del sector.

No ubstante, durante la década de los 80 no se dio la debida importancia al sector hospitalario. Así afirman Newbrander et al".: "Los formuladores de política centralizarán su atención, casi exclusivamente, en la atención primaria de salud aunque fuese sabido que el núcleo de los recursos financieros, de capital y de personal del sistema de salud, se localiza en los hospitales".

En la década de los 90 , se intenta mejorar la eficiencia del sistema de salud y garantizar una utilización mas equitativa de los recursos destinados al sector. Aunque publicado en 1987 el estudio realizado por de Ferranti e colegas (publicado pelo Banco Mundial', 1987)** se situó como un marco

\footnotetext{
* Até agosto de 1994, Asesor del Programa de Desarrollo de Politicas de Salud de la Organización Panamericana de la Salud en la area de Economia de la Salud.

** El estudio Financing Health Services in Developing Countries: An Agenda for Reform fue preparado por John Akin, Nancy Birdsall e David de Ferranti, que en 1987 pertenecian a la División de Politicas y Investigación del Departamento de Población. Salud y Nutrición del Banco Mundial. En este articulo algunas veces me refiero al mismo como el estudio realizado por de Ferranti e colegas. Separatas/Reprints: Antonio Carlos Coelho Campino - Av. Prof. Luciano Gualberto, 908 - 05508-900 - São Paulo, SP - Brasil Fax: (011) 55-11-818-6013.

Recebido en 13.1.1994. Aprobado en 16.3.1995.
} 
en esta area. En este estudio ellos han propuesto el cobro por los servicios (user charges) como una medida que ayudaría a mejorar la alocacion de recursos, mejoraría la eficiencia interna del sector salud y tornaría menor la inequidad en la prestación de servicios de salud.

La tesis central de este trabajo es que la magnitud del déficit de recursos en el sector salud no se puede cubrir a través de medidas como esta propuesta por de Ferranti e colegas. Es necesario recorrer a la vía impositiva. A continuación, se presenta la lógica económica del cobro por la prestación de servicios y se indica los resultados de algunos estudios que han revisado la experiencia de cobro por la prestación de servicios (sección 2). Se presentan algunos ejemplos de acciones adoptadas por algunos países con el objetivo de aumentar la eficiencia del sector salud (sección 3). En la sección 4 se demuestra que hay la posibilidad de aumentar los recursos disponibles para el sector de salud por medio del aumento en la recaudación de impuestos, especialmente en los países pobres de la región y que se puede aumentar la parcela de los ingresos del gobierno que se destiñan al sector salud. Finalmente, se indica como fuentes adicionales de recursos -especialmente para financiar inversión en el sistema de salud en los próximos años- los fondos de emergencia social y los cambios de deuda por salud (sección 5). Estas dos alternativas son parte de la inversión internacional en los sectores sociales.

\section{Cobro por la Prestaclón de Servicios}

La lógica económica para la introducción del pago por servicio es que corresponde a un aumento de precios y por lo tanto reduce la demanda por los servicios de salud que se cobra, incluyendo los que ofrece el hospital.

El pago por servicios ha sido justificado en têrminos de aumento de ingresos, y de promover mayor eficiencia y equidad. Las ganancias en eficiencia serían consecuencia de una mejor utilización de los servicios de salud en general y los hospitalarios en particular. En lo que se refiere a la equidad, se admite que el pago por servicios puede tener efectos positivos y negativos, siendo el efecto neto el resultado de estas dos fuerzas, dependiendo de la manera en que se cobre. Por ejemplo, se puede usar subsidios para aumentar la utilización por los grupos de bajos ingresos. Al mismo tiempo, estos subsidios corrigen elementos regresivos en el sistema actual de pago por servi- cio y en la concentración de clínicas y equipo, incluyendo el hospital. Por otra parte, hay la posibilidad de que cualquier sistema de pago por servicios crea barrieras al acceso de servicios por los más pobres.

Una revisión de las experiencias de los países en desarrollo con el pago de los servicios indica que no es lo suficientemente significante para introducirlo. En el caso de los hospitales, los recursos obtenidos no parecen significante tanto en los hospitales públicos como en los privados. En cuanto a los hospitales públicos, un estudio de Lewis $^{8}(1990)$ sobre tres países latinoamericanos indica que la proporción del presupuesto corriente que era cubierto por la recaudación del pago por servicios variaba del 1 al $8.5 \%$.

En el caso de los hospitales privados esta proporción es mas significante. En el caso de los hospitales mantenidos por misiones en Africa, la proporción de los costos recurrentes recuperada variaba entre el 27 y el $70 \%$. Pero estos hospitales representan solamente una baja proporción de la prestación total de servicios y de los recursos necesarios en el sistema (Newbrander et $\mathrm{al}^{9}, 1992$ ).

Esta insignificancia en impacto del pago por servicios puede tener varias causas. En el caso de los hospitales públicos, el bajo valor obtenido a través del pago por servicios puede ser consecuencia de que el valor cobrado frecuentemente tiene que ser entregado al Gobiemo Central o local y por lo tanto los administradores de los hospitales no tienen ningún incentivo en mejorar sus esquemas de pago. Otra posible causa, tanto para los hospitales públicos como para los privados, puede ser la política de precios, es decir, el hecho de que los precios cobrados solo representan un pequeño porcentaje de los costos reales. Por ejemplo, los programas de pago no se ajustan con la frecuencia necesaria para tener en cuenta la inflación. Otra razón puede ser la práctica de una política de los gobiernos en que se excluye a demasiadas personas de la obligación de pagar. Siempre que no se define claramente quien debe y quien no debe pagar, el número de exclusiones será excesivo.

De cualquier forma, está claro que el pago por servicios debe ser precedido por una mejora en la calidad de los servicios prestados en los hospitales públicos. El Banco Mundial, que es la institución que más ha promovido el pago por servicios, insistía hace cinco años que "el pago por servicios no sería efectivo en aumentar los ingresos a menos que incentivos competitivos... orienten el sistema hacia la provisión de atención de calidad a precios razo- 
nables." (Banco Mundial', 1987).* Newbrander et al ${ }^{9}$., observan que el hecho de que en Africa las personas estaban dispuestas a pagar el costo mayor de los hospitales de misiones, puede estar asociado con la percepción de que la calidad de la atención sea mejor que la de los hospitales públicos.

\section{Mejoras en Eficiencia}

Una forma de aumentar la prestación de servicios de salud con los mismos recursos disponibles es por medio de la mejora en eficiencia en la prestación de los servicios.

Para discutir las posibilidades de aumento de la eficiencia en la prestación de servicios en la área médica es conveniente discriminar entre la eficiencia económica y la eficiencia técnica.**

Un proceso técnicamente eficiente no será económicamente eficiente si los insumos utilizados no son los del menor costo. Como nota Newbrander et al'. (1992), la eficiencia económica se puede aumentar por medio de la substitución de los insumos utilizados o por una mejora en las técnicas de administración. Observase que esto es un campo en la cual el rol del administrador hospitalario es crítico.

En lo que se refiere a la substitución de insumos, Barnum y Kutzin ${ }^{4}$ (1992), presentan como ejemplo la substitución de médicos por enfermeras en determinadas tareas.

La ineficiencia técnica puede resultar en la faita o el uso impropiado de los insumos en los hospitales, personal, suministros y equipo médico. Algunos ejemplos ilustrativos son proporcionados por Newbrander et al'. En cuanto a personal es posible que esquemas de asignación rígida de personal resulten en una ineficiencia técnica. En lo que se refiere a equipamiento, un estudio realizado en China indica que equipamiento diagnóstico (CAT scanners) y equipamiento de tratamiento

\footnotetext{
* Traduccion libre. El texto en ingles dice: "Charging fees at government facilities will not be effective in raising revenue unless competitive incentives in both the nongovernment sector and the decentralized government sector orient the system toward providing quality care at affordable prices" (subrayado por $m i$ ).

** Para el lector no economista, se reproducen los conceptos de eficencia técnica y económica, presentados en Fisher et alt. 1991 .

"Un método de producción es técnicamente eficiente si no existe ningún otro método que utilice una cantidade menor de, al menos, un factor y ninguna más de otro para obtener un nivel dado de producción. La eficiencia técnica es necesária. pero eso no significa que se produzca al menor costo posible (eficiencia económica),"
}

(equipos de diálisis y de la unidad de terapia intensiva) estaban subutilizados (Newbrander et al. ${ }^{9}$ ). Desafortunadamente, hoy en día, muchos países tienen hospitales con equipamiento donado que es inapropiado debido al nivel de servicios que ofrecen, la falta de recursos humanos, o la falta de finanzas para mantenerlo. En cuanto a los medicamentos, ineficiencia pueda ocurrir tanto por error en el tipo de medicamento prescrito así como por error en las cantidades prescritas. Adicionalmente, ocurren perdidas por desvío de drogas y almacenaje en condiciones impropias, o por procedimientos de compra errados. En Mali una reducción del $40 \%$ en el gasto de medicamentos fueron posibles por medio de mejores procedimientos administrativos (Organización Mundial de la Salud ${ }^{10}, 1990$ ).

En nuestra región, la Organización Panamericana de Salud (OPS) ha apoyado estudios en estas áreas. Se han apoyado estudios en eficiencia económica que objectivan determinar costos por patología en Brasil y Cuba. La implementación de esta metodología permite a los directores de los hospitales un acompañamiento constante de los factores de evolución de costos y actuar rápidamente en conjunto con los responsables por las áreas clínicas en la corrección de posibles distorsiones.

Los medicamentos son una parte de los costos de atención de la salud que han estado aumentando en el mundo desarrollado y en el mundo en desarrollo por igual. La elasticidad de los gastos en medicinas con respecto al ingreso se estimó en 1,276 (Gertler y van der Gaag7, 1985); en consecuencia, cuando el ingreso aumenta $1 \%$, los gastos en medicinas aumentarían $1,28 \%$. Estas estimaciones se determinaron sobre la base del ingreso y los gastos familiares, pero podemos suponer que Ios gastos públicos en medicinas también demuestran una elasticidad de ingreso mayor que uno. Las ganancias de eficiencia en el uso de los medicamentos pueden ser una fuente importante de recursos adicionales para otras áreas del sistema de atención médica. Varias iniciativas están teniendo lugar en esta área. De importancia para el sector público es el uso de las medicinas genéricas en los hospitales públicos (cuando están disponibles), y las ganancias en eficiencia en el proceso de adquisición y distribución de los medicamentos. Además, se ha probado que el mejoramiento en el control de dispensión para pacientes internados y ambulatorios y la racionalización en el uso de antibióticos al nivel hospitalario reducen los gastos en medicinas en Cuba. En esta labor, el gobiemo de Cuba recebió ayuda de la OPS. 


\section{Aumento de los Gastos del Gobierno en Salud}

Dada la difícil situación económica con que se enfrentan los países de la región y la necesidad de recuperar urgentemente el ritmo de la inversión y el gasto en salud se plantea la pregunta: $i$ Cómo recuperarán los países de la Región su capacidad de gastar e invertir en salud? La pregunta puede dividirse en dos: Primero, ¿cuánto pueden aumentar los gastos del gobiemo? $\mathrm{y}$, segundo, ¿cuánto puede aumentar la proporción destinada para el sector salud? La respuesta a la primera pregunta sugiere que examinemos cuál es el tamaño relativo del sector del gobiemo en América Latina y el Caribe. Esto puede determinarse mediante el análisis de las cifras de los ingresos y los gastos del gobiemo central. Los datos se presentan en la Tablas 1 y 2 .

Tabla 1 - Gastos e ingresos del gobiemo como proporción del PNB en América Latina y en los paises de la OCDE, 1988.

\begin{tabular}{ccc}
\hline Elemento & $\begin{array}{c}\text { Amérlca Latina } \\
\text { y el Caribe } \\
(\% \text { del PNB })\end{array}$ & OCDE \\
& $(\%$ def PNB $)$
\end{tabular}

\begin{tabular}{lrr}
\hline Ingresos totales (R) & 25,1 & 26,1 \\
Seguridad social (S) & 3,6 & 8,6 \\
Ingresos fiscales (T) & 21,4 & 17,5 \\
Gastos del Gobierno (G) & 23,0 & 29,8 \\
Gastos en salud (H) & 1,4 & 2,7 \\
\hline
\end{tabular}

Obs:: OCDE - Organización de Cooperación y Desarrollo Económico.

Fuente:Banco Mundial ${ }^{2}$ (1990) - Informe sobre el Desarrollo Mundial - Tablas 11 y 12.

Comenzando con los datos sobre los ingresos actuales del gobierno central, se observa que, en promedio, los ingresos fueron $25 \%$ del PNB en la Región. Es decir, en impuestos y contribuciones al seguro social los países de la Región estaban recaudando un cuarto de su producto nacional bruto.

Esto nos llevaría a la conclusión de que por promedio no es mucho lo que los países de la Región pueden hacer para aumentar sus ingresos totales para financiar sus gastos de salud. Naturalmente, esta cifra promedio oculta las grandes diferencias entre países. De los datos de la Tabla 2 se puede ver que, en general, todos los paŕses con ingresos per cápita inferiores a US $\$ 1,300$ pueden aumentar significativamente su tasa de recaudación fiscal puesto que sus ingresos actuales como proporción del PNB están bien por debajo del promedio regional (con excepción de Nicaragua en 1986). México, a pesar de su PNB más elevado, es tambiên un país que puede aumentar sig- nificativamente su recaudación de ingresos actuales para financiar no solo el sector salud sino también otros sectores sociales. En varios de los países de América Latina y el Caribe la recaudación del impuesto a la renta representaba en 1988 una fracción muy pequeña de ingresos actuales totales: $2,7 \%$ en Bolivia, $13,8 \%$ en el Paraguay, $12,7 \%$ en Costa Rica y $11,5 \%$ en el Brasil. Está claro que debe implantarse un sistema más progresivo de tributación.

Las cifras de los ingresos corrientes de los gobiemos centrales incorporan las contribuciones al seguro social, que ascendieron al $3,65 \%$ del PNB de la Región (cuadro 1). Cuando inicialmente consideramos la distribución de las contribuciones al seguro social como porcentaje del PNB, observamos un patrón similar al descrito anteriormente. Los países con ingreso per cápita inferior a US $\$ 1,500$ contribuyen menos que el promedio regional, que se eleva debido a las cifras altas correspondientes a Costa Rica, Panamá, Brasil, Unuguay y Argentina. México y Venezuela también contribuyen porcentajes muy bajos de su PNB, dados sus niveles de PNB per cápita.

Ingresos fiscales es el término que emplearé al referime a los ingresos corrientes del gobierno central cuando se resta el seguro social. En promedio, en América Latina y el Caribe los ingresos fiscales representaron un $21,4 \%$ del PNB. Nuevamente se observa que países con un bajo nivel del PNB per cápita presentan insuficiente recaudación relativa al PNB cuando se compara con el promedio regional. Esto es también el caso en Costa Rica, México, Uruguay y Argentina.

Para el grupo de 14 países de la Región, en 1988 , los gastos del gobierno central ascendieron a $23 \%$ del PNB de estos países, correspondiendo a US $\$ 175.16$ millones (Tabla 2). De nuevo, todos los países con $\mathrm{PNB}$ per cápita iguales o inferiores a US $\$ 1,300$ muestran proporciones bien por debajo de ese nivel. Aun los gobiemos de los países más ricos de la muestra, Argentina y Venezuela, gastaron ligeramente menos que el nivel regional.

Los gastos de salud fueron US\$10.900 millones, correspondiendo a un $6,55 \%$ de todo el dinero gastado por el gobierno central o un $1,43 \%$ del PNB. Observamos nuevamente que los mismos países que necesitan aumentar sus gastos en salud tienen que aumentar sus gastos generales y mejorar su base fiscal.

En conclusión, los datos de las Tablas 1 y 2 indican claramente que los países más pobres de la Región, con un PNB per cápita inferior a US $\$ 1,300$, pueden aumentar la cantidad total de recursos para los sectores sociales en general y la salud en parti- 
Tabla 2 - Características Claves Económicas Relevantes al Sector Salud en América Latina y el Caribe.

\begin{tabular}{|c|c|c|c|c|c|c|c|}
\hline País & $\begin{array}{l}\text { PNB } 1988 \\
\text { US\$ millones }\end{array}$ & $\begin{array}{c}\text { PNB per } \\
\text { cápita } 1988 \\
\text { US\$ }\end{array}$ & $\begin{array}{l}\text { Gasto total } \\
\text { Gobiemo } \\
\text { (\% PNB) }\end{array}$ & $\begin{array}{l}\text { Gasto Salud } \\
\text { Gobierno \% Total } \\
\text { Gasto Gobierno }\end{array}$ & $\begin{array}{l}\text { Ingreso } \\
\text { Total } \\
\% \text { PNB }\end{array}$ & $\begin{array}{l}\text { Seguro } \\
\text { Social } \\
\% \text { PNB }\end{array}$ & $\begin{array}{l}\text { Ingreso* de } \\
\text { Impuesto } \\
\% \text { PNB }\end{array}$ \\
\hline Haití & 2,394 & - & - & - & $10,8 \%$ & $0,0 \%$ & $10,8 \%$ \\
\hline Botivia & 3,933 & 570 & $15,8 \%$ & $1,9 \%$ & $11,8 \%$ & $1,1 \%$ & $10,7 \%$ \\
\hline Pepública Dominicana & $4,96 \mathrm{~B}$ & 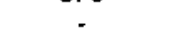 & 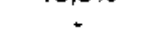 & - & $16,4 \%$ & $0,7 \%$ & $15,7 \%$ \\
\hline Nicaragua & 2,844 & - & - & - & $40,7 \%$ & $5,0 \%$ & $35,7 \%$ \\
\hline Guatemala & 7,830 & 900 & $12,1 \%$ & - & $10,3 \%$ & $0,0 \%$ & $10,3 \%$ \\
\hline El Salvador & 4,700 & 940 & $11,3 \%$ & $7,1 \%$ & $10,5 \%$ & $0,0 \%$ & $10,5 \%$ \\
\hline Ecuador & 11,312 & 1,120 & $14,7 \%$ & - & $14,2 \%$ & $0,0 \%$ & $\uparrow 4,2 \%$ \\
\hline Paraguay & 4,720 & - & - & - & $10,6 \%$ & $1,4 \%$ & $9,2 \%$ \\
\hline Colombia & 37,406 & 1,180 & $17,1 \%$ & . & $13,8 \%$ & $1,2 \%$ & $12,6 \%$ \\
\hline Perú & 26,910 & 1,300 & $14,6 \%$ & $5,8 \%$ & $9,0 \%$ & $0,0 \%$ & $9,0 \%$ \\
\hline Chile & 19,328 & 1,510 & $33,4 \%$ & $6,3 \%$ & $31,7 \%$ & $1,8 \%$ & $29,9 \%$ \\
\hline Costa Rica & 4,563 & 1,690 & $28,0 \%$ & $19,3 \%$ & $22,3 \%$ & $5,9 \%$ & $16,4 \%$ \\
\hline México & 147,312 & 1,760 & $27,9 \%$ & $1,1 \%$ & $18,0 \%$ & $2,1 \%$ & $15,9 \%$ \\
\hline Panamá & 4,876 & 2,120 & $34,4 \%$ & $16,7 \%$ & $31,9 \%$ & $7,9 \%$ & $24,0 \%$ \\
\hline Brasil & 311,904 & 2,160 & $25,1 \%$ & $9,5 \%$ & $34,4 \%$ & $5,7 \%$ & $28,7 \%$ \\
\hline Uruguay & 7,657 & 2,470 & $23,7 \%$ & $4,8 \%$ & $22,1 \%$ & $5,7 \%$ & $16,4 \%$ \\
\hline Argentina & 79,380 & 2,520 & $21,6 \%$ & $2,1 \%$ & $19,7 \%$ & $5,2 \%$ & $14,5 \%$ \\
\hline Venezuela & 61,100 & 3,250 & $21,8 \%$ & $10,0 \%$ & $28,2 \%$ & $0,9 \%$ & $27,3 \%$ \\
\hline América Latir & 761,024 & 1,840 & $23,0 \%$ & $6,2 \%$ & $25,0 \%$ & $3,6 \%$ & $21,4 \%$ \\
\hline
\end{tabular}

* Ingreso de Impuesto = Ingreso Total - Ingreso Seguro Social.

Fuente: Banco Mundial ${ }^{2}$ (1990) - Intorme sobre el Desarrollo Mundial, Tablas 11 y 12.

cular al aumentar su base fiscal, su recaudación fiscal y los gastos del gobierno. Naturalmente, es esencial que toda ganancia en los ingresos se dirija a los sectores sociales. Entre los países de la Región, México tiene claramente mucho por mejorar en todos los parámetros que consideramos, y Costa Rica, Uruguay, Venezuela y Argentina pueden mejorar uno u otro parámetro.*

Una alternativa complementaría que no excluya una ampliación fiscal general que aumente la capacidad del gobiemo, es dirigir una proporción mayor de recursos al sector salud. Pero, los programas del sector salud deben ser sometidos a una continua evaluación con el objetivo de maximizar su eficiencia y eficacia.**

\footnotetext{
* Con relación al PNB, los siguientes paises estuvieron por debajo del promedio regional:

- en los ingresos corrientes: Mexico

- en los ingresos de seguro social: México y Venezuela

- en los ingresos fiscales: México, Costa Rica, Uruguay y Argentina.

** Se esclarece el sentido en que los terminos eficiencia y eficacia son utilizados en este texto. El signficado de eficiencia fue el presentado en Fisher et af. Un programa de salud sera economicamente eficiente se proveer los servicios al menor costo posible: pero esto no significa que será efectivo. Un programa efectivo es aquel que atinge una determinada unidad de objectivo al menor costo possible. Un ejemplo talvez ayude a clarificar como estoy utilizando los dos conceptos. Un programa de desayuno escolar podrá ser el más eficiente (en relacion a otros programas) si tuvier el menor costo por desayuno distribuido, pero puede no ser el más efectivo si no presentar el menor costo por unidad de objectivo (por ejemplo costo por $10 \%$ de reducción en el número de niños con desnutrición de ler grado).
}

En este aspecto hay una gran variación entre los países de la Región. Como se verifica de la Tabla 2, Costa Rica y Panamá tenían la proporción más alta de sus Presupuestos dedicada a la salud (19 y $16 \%$ ), el Brasil y El Salvador ocuparon una posición intermedia $(9,5$ y $7,1 \%)$, y Perú y Chile estuvieron cerca del promedio. Lo que los datos ilustran claramente es que los países varían en la importancia que atribuyen al sector salud, un parámetro político que cada gobieno puede manipular. México y Argentina pueden aumentar sustancialmente sus gastos en salud como proporción del gasto total del gobiemo para alcanzar el promedio regional, y Uruguay está algo debajo del nivel regional.

\section{Medios Alternativos de Flnanciación del Sector Salud}

Hay dos fuentes alternativas de fondos para el sector salud, las cuales podrían ayudar considerablemente a cerrar las brechas en el nivel de los servicios prestados por el sector debido a la crisis del último decenio, los fondos sociales de emergencia o fondos de inversión social (FSE-FIS) y la conversión de deuda por salud.

\section{Fondos Sociales de Emergencia}

La OPS preparó un documento que evaluó las implicaciones sobre el sector salud, de los fondos 
sociales de emergencia (FSE) y de los fondos de inversión social (FIS) (OPS/OMS ${ }^{\prime}, 1992$ ). Sus objetivos eran la identificación de los objetivos, la determinación del tipo de programas que financiaron y los problemas relacionados con su ejecución institucional. El cuadro 3 presenta un resumen de los países y los programas.*
La evaluación de los programas ya mencionados financiados mediante los fondos sociales de emergencia indica la existencia de problemas en la sostenibilidad, focalización, eficiencia, equidad, organización institucional, vigilancia y evaluación. Cada uno de estos aspectos se examinará brevemente a continuación.

Tabla 3 - Tamaño relativo de las proyecciones del Fondo Emergencia/Inversión Social en Salud.

\begin{tabular}{|c|c|c|c|c|c|}
\hline País & $\begin{array}{l}\text { Salud-FSE } \\
\text { millones }\end{array}$ & Años & $\begin{array}{c}\text { Salud-FSE/Año } \\
\text { US\$ millones }\end{array}$ & $\begin{array}{c}\text { Salud Total/Año } \\
\text { US\$ millones }\end{array}$ & $\begin{array}{c}\text { Salud-FSE/Salud } \\
\text { Total } \%\end{array}$ \\
\hline $\begin{array}{l}\text { Bolivia a/ } \\
\text { Costa Rica } \\
\text { Guatemala } \\
\text { d/ } \\
\text { Honduras } \\
\text { Jamaica } \\
\text { Nicaragua } \\
\text { Perú ol }\end{array}$ & $\begin{array}{c}37,6 \\
3,19 \\
\text { N.A. } \\
6,8 \\
25,7 \\
1,5 \text { al } \\
44,8 \\
29,0\end{array}$ & $\begin{array}{c}87-91 \\
83-84 \\
90 \\
91-93 \\
90-95 \\
\text { N.A. } \\
90\end{array}$ & $\begin{array}{c}9.4 \\
1.6 \\
\text { N.A. } \\
2.3 \\
4.3 \\
\dot{2} .0\end{array}$ & $\begin{array}{c}11.8 \\
246.4 \\
57.9 \\
94.1 \\
53.2 \\
333.84 \text { b/ } \\
227.9\end{array}$ & $\begin{array}{c}79,7 \\
0,6 \\
\text { N.A. } \\
2,4 \\
8,0 \\
\text { N.A. } \\
12,7\end{array}$ \\
\hline
\end{tabular}

a) 1987 a Diciembre de $1990=4$ anōs

b) En base de gastos reportados de US\$ 90 por persona por año, en "Condiciones de Salud en las Américas".

c) Agosto a Diciembre de 1990.

d) Este programa fue discontinuado con la entrada de un nuevo gobierno.

Fuentes: OPS/OMS"1 (1992)

OPS/OMS ${ }^{12}(1990)$

Banco Mundial² (1990)

Una posible manera de evaluar la importancia relativa de los programas de FSE en la salud es comparar la cantidad en dólares que estos programas gastaron en relación con el presupuesto general del sector salud, que es lo que hice en la Tabla 3.

Puede verse que, anualmente, los montos, gastados por los FSE en la salud variaron entre US\$1.6 millones en Costa Rica, a US\$29.0 millones en Perú. Si sacamos el caso excepcional del Perú, donde el FSE duró solo 6 meses, el único país que asignó una cantidad significante del FSE a la salud fue Bolivia (Tabla 3).

También puede observarse en el cuadro 3 que el gasto del FSE fue significante cuando se lo comparó con el gasto total del gobierno central en salud solo en los casos de Jamaica $(8,1 \%)$, Perú (12\%, aunque solo durante un período corto) y Bolivia $(79,6 \%)$. También muestra por qué los FSE pueden tener una repercusion importante, como en el caso de Bolivia, donde representan una casi duplicación de los fondos normales disponibles para el sector salud.

* Al lector interesado en la evaluación de la repercusión de los fondos sociales de emergencia en el sector salud se le remite a tos estudios realizados por OPS/OMS "(1992), Suárez" (1992) y Campinos (1992). a) Con respecto a la sostenibilidad la experiencia de Bolivia indica la importancia de una evaluación de la capacidad financiera de las instituciones beneficiarias y las comunidades.

b) La eficacia de la mayoría de los programas podría mejorarse si se destinan a grupos específicos mediante los mapas de pobreza con que se cuenta para la mayoría de los países de la Región. Los datos recogidos en las evaluaciones de los países indicaron que solo en el caso de Costa Rica los programas fueron focalizados hacia los segmentos más pobres de la población.

c) En la ejecución de los FSE ha habido más énfasis en la eficiencia que en la equidad o la eficacia. Las cuestiones de equidad se relacionan con el hecho de que estos programas tenían objetivos de corto plazo (Suárez ${ }^{13}, 1992$ ). La selección de proyectos de pequeña escala con alta visibilidad política que pueden ejecutarse rápidamente permite un desembolso rápido de los recursos pero no garantiza que los beneficios del proyecto lleguen a los más necesitados, ni que los proyectos tengan tasas de rendimiento social más altas (Suárez ${ }^{\text {t3 }}, 1992$ ).

d) Los programas se enfrentaron con un obstácuto sustancial cuando intentaron evaluar sus "resultados" porque desde el comienzo no se 
habían definido claramente ni los objetivos $n i$ los indicadores que se utilizarian.

e) En lo que se refiere a la base institucional, en general los FSE han obviado las estructuras tradicionales del sector público, excepto en el caso de Costa Rica, donde el fondo fue administrado por la Comisión Nacional de Emergencia. El análisis de Pollack y Wurgaft ${ }^{12}$ de la experiencia boliviana recalca que el FSE no fue parte de una política social integral y solo respondió a las demandas generadas por el ajuste estructural. Como resultado, no se había establecido un orden de prioridad entre las nuevas, pero sustanciales, necesidades de las poblaciones de bajos ingresos y pauperizados (Pollack y Wurgaft' ${ }^{12}, 1990$ ).

f) La estrategia a corto plazo de los FSE no aborda el problema más crucial en muchos de estos países: cómo aumentar la eficiencia y la eficacia del sector público.

Una cuestión final que debe considerarse es que si los FSE representaron una cantidad importante de recursos solo en el caso de tres países (Bolivia, Perú y Jamaica) ¿Por qué se les han atribuido tanta importancia? En mi opinión, la respuesta reside en su flexibilidad de manejo y en el hecho de que probablemente en su caso la razón entre los costos del proyecto y los costos totales es significativamente mayor que en el sector público tradicional, en el que una cantidad considerable de recursos se destina a los salarios y a los gastos generales. Por lo tanto, en términos de edificios, empleo y generación de ingresos el FSE en Jamaica pudo haber producido mucho más de lo que su presupuesto Ilevaría a creer, que fue igual a solo $8 \%$ del presupuesto del Ministerio de Salud.

En conclusión, los FSE pueden representar una cantidad significante de recursos para el sector salud. Si estos recursos se gastaran en forma acertada, podrían ejercer una influencia importante en la obtención de recursos para el sector y podrían financiar nuevos e innovadores conceptos en la prestación de servicios de salud. Con el fín de lograr estos resultados las autoridades de salud tienen que aumentar su eficiencia en la preparación de programas con antelación, en el establecimiento de criterios para la selección de proyectos y programas (por ejemplo, sostenibilidad y fijación de metas), y en la definición de prioridades de inversión e indicadores de evaluación. Los organismos internacionales deben apoyar a los Ministros de Salud de la Región proporcionándoles asistencia técnica para aumentar su capaci- dad para generar proyectos aptos de fínanciamiento mediante los FSE.

\section{Conversión de Deuda por Salud}

Otra potencial fuente de recursos adicionales que la OPS ha estudiado y evaluado es la conversión de deuda por recursos de salud. Si bien hay numerosas variaciones de este concepto, la transacción básica sigue siendo la misma: el intercambio de las obligaciones pendientes del gobierno por otras obligaciones, por lo general denominadas en la moneda del país en lugar de serlo en divisas. El gobierno se compromete a invertir el equivalente en moneda local en proyectos prioritarios de salud. Debido a que la deuda de muchos países se sigue comerciando bien por debajo del valor par en el mercado secundario, fondos del proyecto son obtenidos localmente cuando se pagan a un precio mayor que en el mercado secundario. En último término, pero no menos importante, los países deudores pueden amortizar parte de su deuda restante y revertir la salida de capital que caracterizó los últimos años de la década de los ochenta.

Con respecto a la eficiencia, las conversiones de la deuda también tienen ventajas. Por ejemplo, los donantes y las organizaciones colaboradoras pueden seleccionar los proyectos para financiar mediante conversiones que incluyen planes de continuidad, fijación de metas y evaluación. En forma similar, las conversiones de deuda pueder especificar que los adjudicatarios de la moneda local sean organizaciones no gubernamentales, con lo cual se ayudaría a fortalecer la capacidad voluntaria privada local y a profundizar la red de servicios de salud.

Sin embargo, las conversiones de deuda no han dejado de tener oposición. Ha aumentado la resistencia al uso de este mecanismo debido a la creencia de que estas conversiones pueden tener una repercusión inflacionaria perjudicial en el país deudor. Sin embargo, dado que la mayoría de los proyectos de salud incluyen una cantidad pequeña de recursos en relación con el PNB, es dudoso que puedan tener una repercusión inflacionaria sustancial. Además, los bancos centrales tienen control sobre estas presiones. Mientras el UNICEF (Fondo de las Naciones Unidas para la Infancia) está promoviendo las conversiones de la deuda y la OPS ha invertido una cantidad importante de recursos en la recolección de información, muchas de las otras instituciones multilaterales han perdido su entusiasmo inicial. La OPS está preparada 
para proporcionar asistencia técnica a los países interesados en esta modalidad.

Hay un gran número de actores y variables que intervienen en la realización de una conversión de deuda, y el camino desde la negociación hasta la ejecución no es parejo. Sin embargo, si hay una escasez de asistencia para el desarrollo dirigida hacia el sector salud, las conversiones de la deuda pueden ser una opción cada vez más importante para la Región.

\section{Conclusión}

Está cliaro que el sector salud debe preocuparse por adquirir nuevos recursos tanto nacionales como internacionales en el futuro cercano. La década de los ochenta fue de profunda transformación y de una crisis cuyas consecuencias todavía no se han manifestado plenamente. Es posible que si hubo una regresión en el estado de salud de las poblaciones de la Región, quizás no experimentemos sus efectos totales hasta más adelante en esta década. Lo más importante, es cuando la crisis afectó a la Región, la meta de salud para todos en el año 2000, declarada en Alma-Ata en 1978, no se había logrado todavía. Hoy, la presión demográfi-

\section{Referencias Blbllográficas}

1. BANCO MUNDIAL. Financing bealth services in developing countries: an agenda for reform. Washington,D.C., Banco Mundial, 1987.

2. BANCO MLNDLAL. Informe sobre el desarrollo mundial 1990: pobreza. Washington, D.C., Oxford University Press, 1990.

3. BANCO MUNDLAL. Informe sobre el desarrollo mundial 1991: La tarea acuciante del desarrollo. Washington, D.C., Oxford Lniversity Press, 1991

4. BARNEM, H. \& KLTZIN, J. Public bospitals in developing countries: resourse use, costs, financing. Baltimore, Johns Hopkins Lniversity Press, 1992.

5. CAMPINO, A.C.C. Social emergency funds and investment funds in Latin America and the Caribbean. In: World Health Organization. Macroeconomic environment and bealtb with case studies for countries in greatest need. Geneva, 1993.p.119-36.

6. FISCHER, S. et al. Economia. Madrid, McGraw Hill, 1991.

7. GERTLER, P. \& VAN DER GAAG, J. The willingness to pay for medical care:evidence from ca constante y las epidemias como el SIDA y el cólera son tan solo dos razones por las que debemos renovar nuestro compromiso con las metas básicas formuladas en Alma-Ata. Además, son esos los objetivos que continúan siendo la prioridad de los países que contribuyen con la ayuda exterior para las actividades de salud.

Según lo indican los datos sobre gastos e ingresos del gobiemo central en los países de la Region, es importante que los gobiernos se dediquen seriamente a las reformas que les permitirán invertir productivamente en los sectores sociales. Los recursos nacionales e internacionales deben complementarse para que las economías de América Latina y el Caribe vuelvan a sumarse al proceso de desarrollo. Hay poca duda de que el modelo altamente competitivo de desarrollo dirigido hacia la exportación, que está promoviéndose y prosiguiéndose en todo el mundo, requiere una fuerza laboral más saludable, mejor educada, y más participatoria.

\section{Agradecimlento}

A la OPAS para el apoyo recebido asi como los comentarios de los revisores anónimos.

two developing countries. Washington, D.C.,The World Bank/Johns Hopkins University Press, 1990.

8. LEWIS, M. Cost recovery in developing country bospitals: the determinants of use fee revenues. Washington, D.C., The Urban Institute 1990. (Working Paper 2201-01).

9. NEWBRANDER, W. et al. Hospital economics and financing in developing countries. Geneva, World Health Organization, 1992.

10. ORGANIZACIÓN PANAMERICANA DE LA SALUD. Programa de Desarrollo de Políticas de Salud. Fondos y programas de compensación social: experiencias en América Latina y el Caribe: Washington, D. C., 1992.

11. ORGANIZACIÓN PANAMERICANA DE LA SALLD, Las condiciones de Salud en las Américas, Washington, D.C., 1990.

12. POLLACK, M. \& WLRGAFT J. Programas de generación de empleo y el objetivo ocupacional de los fondos de desarrollo social. In: Seminario Internacional sobre Fondos de Desarrollo Social, Santiago, Chile, 1990. p. 57-110.

13. SUÁREZ, R. Analisis comparativo de los fondos de compensación social. In: Organización 
Panamericana de la Salud. Fondos y programas de compensación soctal experiencias en América Latina y el Caribe. Washington, D.C., 1992
14. WORLD HEALTH ORGANIZACION. Cost analysis in primary bealtb care; a training manual for programme managers. Geneva, WHO/Unicef/Aga Khan Foundation, 1990.

\begin{abstract}
Latin America and the Caribbean ( $L A C$ ) countries are experiencing both an economic crisis and a crisis in the public sector. As a result it is impossible to increase the amount of resources avaliable to the health sector, unless there is a drastic restructuring of the way in which financing occurs. The measures so far referred to in the economic debate user fees, cost recovery, privatization - at best represent partial solutions. Given the magnitude of health problem in LAC countries, they are unable to generate the amount of money needed to cover the deficit of financial resources for medical treatment. The central idea behind this article is that in order to cover the deficit of resources for medical treatment it is necessary to utilize fiscal resources. It is shown that it is possibie to increase the amount of financial resources available for medical treatment either through increases in taxes andor through an increase in the proportion of the government budget dedicated to medical treatment. Increases in taxes collected provide a feasible alternatlve. In some of the poor countries of Latin America and the Caribbean, the proportion of the Gross National Product that goes for the payment of taxes is well below the figure for that proportion found in developed countries. To increase the proportion of the government budget dedicated to medical treatment is a political decision that depends solely upon the discretion of the governments concemed. The potential of Social Emergency Funds and debt swaps to finance innovations in the production of medical treatment services, thus maintaining the current level to activity in the sector, is discussed.
\end{abstract}

Financing, health. Financing, government. Health expenditurs.

\title{
Resumo
}

Dada a magnitude de problema de saúde nos países da América Latina e do Caribe, náo é possível cobrir o déficit de recursos financeiros recessários para prover assisténcia médica através de aumentos nos preços, ou com a adoçāo de medidas como o pagamento por serviços ou a cobrança do custo dos serviços. Faz-se necessário recorrer a politica fiscal. Fol mostrado que é possivel aumentar os recursos disponiveis para assisténcia médica, tanto por meio do aumento na arrecadaçāo de impostos, quanto pela tomada da decisāo politica de aumentar a parcela da receita do Governo, destinaja a financiar a assisténcia médica. Foi revista a experiência dos Fundos de Emerge̊ncia Social e das operaçōes de troca de divida por projetos na area de saúde como importantes fontes adicionais de recursos, especiaimente para financiar o investimento.

Financiamento da saúde. Financiamento govermamental, Gastos em saúde. 